\title{
China's first international HIV vaccine meeting: The war of the roses
}

Although the 30 or so foreign scientists who attended China's first International AIDS Vaccine Workshop at the end of November found little that they had not heard before in their Western colleagues' scientific presentations, they benefited from first-hand reports on China's vaccine production capabilities, and on the status of the epidemic in three areas of China-Yunnan and Sichuan Provinces and Xinjiang Uygur Autonomous Region. They also learned that China is not exempt from the political and egotistical problems that afflict AIDS vaccine research in other countries.

According to Yiming Shao, deputy director of China's National Center for AIDS Prevention and Control (NCAIDS), the workshop was designed to allow exchange of information between foreign and Chinese researchers and assist in focussing the government's attention on the country's increasingly serious HIV problem. Officials estimate that 400,000 people in China are HIV-positive.

Hosted by NCAIDS, the Ministry of Health, the Ministry of Science and Technology and the Chinese Academy of Preventive Medicine, the workshop began in Beijing and ended with a field trip to Yunnan Province in southwestern China, the area hardest hit by the country's growing AIDS epidemic, but also home to a modern vaccine production facility.

But the question of who will be approved to run trials of vaccines, and when, were major subtexts to the meeting, and if not addressed formally, they risk dividing NCAIDS and China's AIDS research efforts.

Reports have surfaced of a potential conflict between two groups of researchers that wish to pursue clinical trials of a clade C HIV vaccine in China (Science 286, 1062; 1999). One group comprises David Ho of the Aaron Diamond AIDS Research Center (ADARC) in New York and former ADARC scientist Yunzhen Cao. Ho anticipates support from the International AIDS Vaccine Initiative (IAVI) for vaccine development. Ho's potential inside track on China includes his many years of working with NCAIDS researchers and with $\mathrm{Cao}$, who, after returning to China in 1998 to run clinical studies on vertical transmission of HIV, was promoted by NCAIDS Director Ke-an Wang to the position of deputy director of NCAIDSequal to Shao.

The other interested party is an EUsupported effort called EuroVac, which includes Hans Wolf from the University of Regensburg, Germany, an AIDS researcher with a long history of collaboration in China, and Shao's mentor. Shao seems to have a foot in both camps, as he is also a member of IAVI's working group for an AIDS vaccine

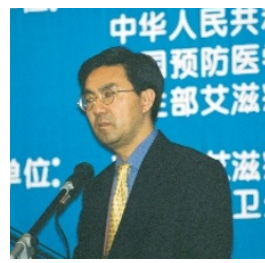

Yiming Shao
However, the point of who gets to test vaccines first is at present moot, because China does not yet have a mechanism for approving such clinical trials. In fact, one of the goals of the workshop was to circulate for comment a draft National Strategic Plan for the Development and Evaluation of HIV/AIDS Vaccines in China. Even with China now indicating that AIDS vaccine candidates will receive accelerated treatment, approval to begin such trials may take several months.

One point of concern emphasized at the meeting, and one that may influence cliniprogram in China. He sits across the hall from Cao at NCAIDS.

Although Shao denied knowledge to Nature Medicine of Ho's wish to set up trials in China until recently, there was a distinct impression at the workshop that some of the people at NCAIDS who are cooperating with each of the projects have contributed to the conflict. But Shao stresses that he sees no clash between the two plans: "If it's good science and a rational design, we should open our minds. Even if that's a parallel project, it should be okay."

Many scientists came away from the workshop with the impression that the conflicting interests within NCAIDS, which may reflect deeper rifts within the Chinese AIDS research establishment, were deep-seated. These rifts were evident on the first day of the workshop, when Shao held an invitation-only meeting with Vice Minister of Health Da-kui Yin, which excluded some of his NCAIDS colleagues.

This was followed by another invitation-only event organized by Cao to mark the signing of

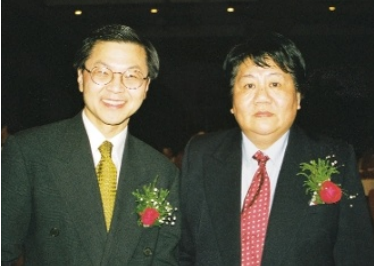

David Ho and Yunzhen Cao cal trial conduct, is the delicate political facets of China's AIDS surveillance program-such as dealings with sex workers, a group likely to be exposed to HIV and to be targeted for vaccine trials but who, if caught by law enforcement authorities, would be forced into government reeducation programs.

On a more positive note, attendees complimented the quality of research and expressed a desire to work with China to develop a vaccine. Peggy Johnston, assistant director for AIDS vaccine development at the National Institute of Allergy and Infectious Diseases, National Institutes of Health (NIH), noted "discussions that started earlier this year could come to fruition soon in the form of a first NIH-China collaboration, which is beyond the training [exchanges] already underway and individual R01 grants."

Johnston was impressed by China's will to carry out the work necessary to find a vaccine and unconcerned by the politics that, she says, are "virtually everywhere" and are not unique to China. But Harvard School of Public Health's Yichen Lu warns, "The power struggle inside Chinese a cooperative agreement between NCAIDS and ADARC, which excluded most non-Chinese at the meeting. Those invited to the latter affair were later distinguishable at dinner by their rose corsages. Unflowered Wolf brushed off the event, explaining that the EU signed a similar, routine agreement with NCAIDS several months ago.
NCAIDS has seriously damaged the image of the center, discouraging muchneeded international funding and collaborations. We are wasting precious time when the AIDS epidemic can still be stopped at the early stages in this country."

Myrna Watanabe, Beijing 